Dr. med. Ulrich Mutschler, Hamburg

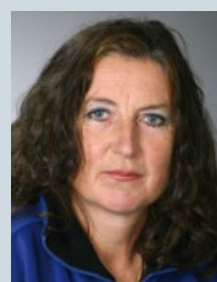

Dr. med. Kirsten Stollhoff, Hamburg $a b$, was nun offensichtlich in Frage gestellt werden muss. Die Studie zeigt, dass Kinder mit atopischer Diathese, die im ersten Lebensjahr kontinuierlich erdnusshaltige Nahrungsmittel bekommen, im Alter von 5 Jahren signifikant seltener an einer Erdnuss- allergie leiden gegenüber Kindern, die dieses Allergen konsequent mieden.

Insgesamt zeichnet sich generell ein komplettes Umdenken in der Primär- und Sekundärprophylaxe von Allergien ab: Weg von der Abstinenz, hin zur frühen Toleranz- entwicklung durch nicht zu spätes Füttern von früher als hochpotent eingeschätzten Nahrungsmittelallergenen wie Fisch und Milch. Eine Exposition, die nicht zu spät kommt, erzeugt eine orale Toleranz.

Dr. Ulrich Mutschler

\title{
Nur wiederholtes Reanimationstraining steigert die Qualität
}

\section{Die Fachgesellschaften unterstützen die flächendeckende Umsetzung des Simulationstrainings als effektive Methode in der Ausbildung zur Herz-Kreislauf-Wiederbelebung. Wie oft sollte diese trainiert werden?}

m Rahmen einer Multicenterstudie aus den USA wurde der Frage nachgegangen in welchen Zeitabständen ein Simulationstraining wiederholt werden sollte. In dieser randomisierten und prospektiven Untersuchung wurden 42 angehende Kinderärzte beobachtet. Sie erlangten im Rahmen eines Basistrainings in vier standardisierten Simulationsszenarien eine definierte geprüfte Qualifikation. Die vier zu bearbeitenden Szenarien hypovolämischer Schock, Asystolie, respiratorisches Versagen und Mekoniumaspiration betrafen jeweils junge Säuglinge und wurden anhand eines computergesteuerten Simulationsbabys durchgeführt und geprüft.

Anschließend erfolgte eine Wiederholungstestung in unterschiedlichen Zeitintervallen. Nach 2 Monaten bestanden $92 \%$, nach 4 Monaten $71 \%$ und nach 6 Monaten nur noch $56 \%$ der Ärzte die im Basistraining geforderte und damals auch erlangte Ausgangsqualifikation.
Braun $L$ et al. Retention of pediatric resuscitation performance after a simulation-based mastery learning session: a multicenter randomized trial. Pediatric Critical Care Medicine $2015 ; 16 ; 131-8$

\section{Kommentar}

Das Ergebnis überrascht nicht. Allerdings war das Ausgangstraining mit nur 1-2 Stunden Dauer reichlich kurz bemessen, das Trainingsteam bestehend aus einem einzelnen zu prüfenden Arzt und einer Pflegekraft war unrealistisch übersichtlich und im Protokoll wurden auch nur harte Fakten wie zeitlich korrekt durchgeführte Perfusionskontrolle oder Sauerstoffverabreichung mit Punkten bewertet. Sogenannte "soft skills" wie Kommunikation im Team fanden offensichtlich keinen Eingang in die Ausbildung beziehungsweise die Re-Testung. Zu fordern ist ein intensiveres Basistraining in realen Teams unmittelbar am Arbeitsplatz - gleichermaßen ob nun in der Klinik oder in der Arztpraxis. Die Wiederholung dieser Trainings muss regel-

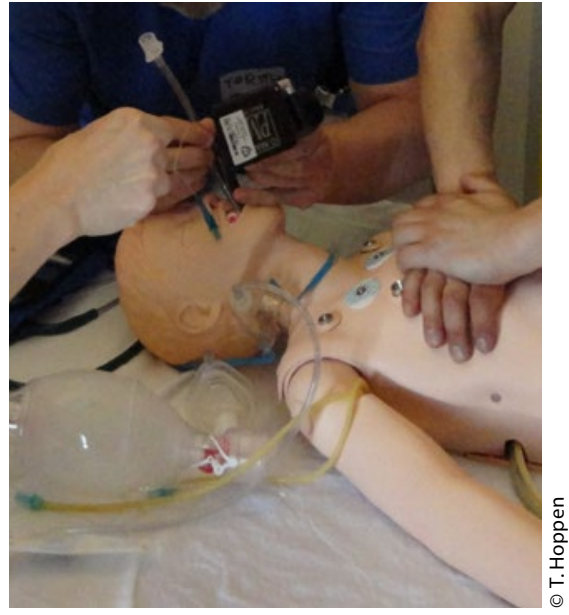

Kindernotfall-Simulationstraining am PC-gesteuerten Mannequin im Team

mäßig erfolgen, zum Beispiel in 3-6 monatlichen Abständen. Fehlende und damit limitierende Faktoren im Alltag sind leider immer noch in vielen Einrichtungen Reanimationsmodelle, kompetente Instruktoren, positive Bereitschaft bei allen Teammitgliedern und die Einsicht, dass diese konkurrenzlose Maßnahme zur qualitätssteigernden Teamentwicklung unumschränkt in die übliche Arbeitszeit verankert gehört. Das Netzwerk Kindersimulation (www.netzwerk-kindersimulation.org, vgl. S. 62 dieser Ausgabe) beschäftigt sich mit diesem Thema. Dr. Thomas Hoppen 